\title{
IFITM3 inhibits virus-triggered induction of type I interferon by mediating autophagosome-dependent degradation of IRF3
}

\author{
Li-Qun Jiang ${ }^{1}$, Tian Xia ${ }^{1}$, Yun-Hong Hu${ }^{1}$, Ming-Shun Sun ${ }^{1}$, Shuang Yan ${ }^{1}$, Cao-Qi Lei ${ }^{1}$, \\ Hong-Bing Shu ${ }^{1}$, Ji-Hua $\mathrm{Guo}^{2}$ and $\mathrm{Yu} \mathrm{Liu}^{1}$
}

Interferon-induced transmembrane protein 3 (IFITM3) is a restriction factor that can be induced by viral infection and interferons (IFNs). It inhibits the entry and replication of many viruses, which are independent of receptor usage but dependent on processes that occur in endosomes. In this study, we demonstrate that IFITM3 plays important roles in regulating the RNA-virus-triggered production of IFN- $\beta$ in a negative-feedback manner. Overexpression of IFITM3 inhibited Sendai virus-triggered induction of IFN- $\beta$, whereas knockdown of IFITM3 had the opposite effect. We also showed that IFITM3 was constitutively associated with IRF3 and regulated the homeostasis of IRF3 by mediating the autophagic degradation of IRF3. These findings suggest a novel inhibitory function of IFITM3 on the RNA-virus-triggered production of type I IFNs and cellular antiviral responses. Cellular \& Molecular Immunology advance online publication, 24 April 2017; doi:10.1038/cmi.2017.15

Keywords: autophagy; IFITM3; IFNs; IRF3

\section{INTRODUCTION}

As the first line of host defense, the innate immune system counters viral infection by expressing a number of intrinsic antiviral proteins, triggering the production of interferons (IFNs) and facilitating the activation of adaptive immunity. Intrinsic antiviral proteins, which are constitutively expressed, directly restrict the invasion or replication of viruses. Most intrinsic antiviral proteins can also be induced by IFNs. The expression of IFNs depends strictly on the recognition of pathogens by endosomal Toll-like receptors (TLRs) or cytosolic viral sensors, such as retinoic acid-inducible gene I (RIG-I) and cyclic GMP-AMP synthase. These receptors trigger the transduction of different signals, leading to the induction of type I IFNs (including IFN- $\alpha$ and IFN- $\beta$ family members) and proinflammatory cytokines. Type I IFNs activate the Janus kinase signal transducer and activator of transcription pathway, and initiate the transcription of IFN-stimulated genes (ISGs). The products of these genes inhibit viral replication, eradicate virus-infected cells and facilitate the activation of antiviral adaptive immunity. ${ }^{1,2}$
TLRs and RIG-I-like receptors (RLRs) detect RNA virus infection. For example, TLR3 recognizes viral double-stranded RNA (dsRNA) released by infected cells and triggers TIRdomain- containing adapter-inducing interferon- $\beta$-mediated signaling pathways, whereas TLR7 and TLR8 recognize viral single-stranded RNA and activate MyD88-dependent signaling. ${ }^{3,4}$ The RLRs, such as RIG-I and MDA5, recognize cytoplasmic viral RNA through their C-terminal RNA helicase domains, and then recruit the downstream adapter protein virus-induced signaling adaptor (VISA, also known as MAVS, IPS-1 and Cardif) through their CARD domains. ${ }^{5-8}$ Both TLRand RLR-triggered signaling cascades activate the downstream kinases TBK1-IKKe and TAK1-IKK $\beta$, leading to the activation of interferon regulatory factors (IRFs) and NF-KB. These activated transcription factors collaboratively trigger the transcription of type I IFN genes. ${ }^{9,10}$

Interferon-induced transmembrane (IFITM) proteins are intrinsic antiviral restriction factors. Like other intrinsic factors, the IFITMs can be further induced by IFNs. ${ }^{11,12}$ IFITM3, a member of the IFITM family, has been extensively studied

${ }^{1}$ State Key Laboratory of Virology, Hubei Key Laboratory of Cell Homeostasis, Department of Cell Biology, College of Life Sciences, Wuhan University, Wuhan 430072, China and ${ }^{2}$ Hubei-MOST KLOS \& KLOBME, School \& Hospital of Stomatology, Wuhan University, Wuhan 430072, China

Correspondence: Dr Y Liu, State Key Laboratory of Virology, Hubei Key Laboratory of Cell Homeostasis, Department of Cell Biology, College of Life Sciences, Wuhan University, Wuhan 430072, China.

E-mail: yuliu@whu.edu.cn

Received: 17 September 2016; Revised: 19 January 2017; Accepted: 12 February 2017 
because it plays an important role in preventing endocytosed viral particles from accessing the host cytoplasm. Many viruses are inhibited by IFITM3, including Dengue virus, West Nile virus, Influenza A virus (IAV), flaviviruses, severe acute respiratory syndrome coronavirus and Vesicular stomatitis virus (VSV). ${ }^{13-15}$ These are all enveloped viruses and enter the cell by endocytosis. It has been reported that IFITM3 restricts viruses independently of their receptor usage but is dependent on processes that occur in the endosome. It prevents membrane hemifusion mediated by envelope proteins of IAV and restricts the early steps of IAV replication. ${ }^{16}$ It also restricts Human immunodeficiency virus 1 (HIV-1) infection by antagonizing the envelope glycoprotein. ${ }^{17}$ IFITM3 restricts the entry of reoviruses, which are nonenveloped viruses, and utilizes endosome-dependent cell entry mechanisms. ${ }^{14}$ Although the role of IFITM3 in antiviral immunity has been investigated intensively, here we report our novel observation that IFITM3 negatively regulates the virus-induced production of type I IFNs. Sendai virus (SeV), which fuses with the cell surface, is reportedly not restricted by IFITM3. ${ }^{18}$ In this study, we demonstrate that IFITM3 negatively regulates $\mathrm{SeV}$-induced production of type I IFNs by targeting IRF3. Thus, we report a distinctive mechanism of IFITM3 in antiviral immunity.

\section{MATERIALS AND METHODS}

\section{Constructs}

$N F-\kappa B$, interferon-sensitive response element (ISRE), IFN- $\beta$ promoter and IRF1 luciferase reporter plasmids and plasmids for human TBK1, IRF3, VISA and RIG-I have been described. ${ }^{19,20}$ The mammalian expression plasmid for human Flag-tagged IFITM3 was a kind gift from Dr Chen Liang at McGill University and Dr Wentao Qiao at Nankai University. The mammalian expression plasmids for human ATG5 and Beclin1 were provided by Dr Rongjia Zhou at Wuhan University. GFP-Newcastle disease virus (NDV) and GFPVSV have been previously described. ${ }^{21}$

\section{Reagents and antibodies}

Poly(I:C) (Invitrogen, Carlsbad, CA, USA), IFN- $\gamma$ (Peprotech, Rocky Hill, NJ, USA), IFN- $\alpha$ (Peprotech), TRIzol Reagent (Takara, Dalian, China), Gamma Bind G Plus-Sepharose (Amersham Biosciences, GE Healthcare, Marlborough, MA, USA) and the human IFN- $\beta$ ELISA kit (PBL, Piscataway, NJ, USA) were purchased from the indicated manufacturers. HEK293 and HeLa cells were obtained from the ATCC. Mouse anti-Flag (Sigma, St Louis, MO, USA), mouse anti- $\beta$-actin (Sigma), mouse anti-GFP (Santa Cruz Biotechnology, Santa Cruz, CA, USA), mouse anti-LMNB1 (Proteintech, Rosemont, IL, USA), rabbit anti-IRF3 (Santa Cruz Biotechnology), rabbit anti-pIRF3 (CST, Danfoss, MA, USA), rabbit anti-VISA (Bethyl, Montgomery, TX, USA), rabbit anti-TBK1 (Abcam, Cambridge, UK), rabbit anti-pTBK1 (Abcam) and rabbit antiLC3 (Sigma) antibodies were purchased from the indicated manufacturers. Rabbit and mouse antibodies against IFITM3 were raised against recombinant human IFITM3.

\section{Transfection and reporter assays}

HEK293 cells or HeLa cells were transfected using the calcium phosphate precipitation method or FuGENE Transfection Reagent (Roche, Basel, Switzerland). The pRL-TK Renilla luciferase reporter plasmid was added to each transfection reaction to normalize for the transfection efficiency. The luciferase assays were performed using the Dual-Luciferase Reporter Assay System (Promega, Madison, WI, USA). ${ }^{22}$

\section{Coimmunoprecipitation and immunoblot analyses}

Transfected HEK293 cells from 10-cm dishes were lysed in $1 \mathrm{ml}$ of Nonidet P-40 lysis buffer (1\% Nonidet P-40, $150 \mathrm{mM} \mathrm{NaCl}$, $1 \mathrm{mM}$ EDTA, 20 mM Tris-HCl, pH 7.4-7.5, $10 \mu \mathrm{g} / \mathrm{ml}$ leupeptin, $10 \mu \mathrm{g} / \mathrm{ml}$ aprotinin and $1 \mathrm{mM}$ phenylmethylsulfonyl fluoride). For each immunoprecipitation reaction, $0.9 \mathrm{ml}$ of the cell lysate was incubated with $25 \mu \mathrm{l}$ of Gamma Bind G PlusSepharose and $0.5 \mu \mathrm{g}$ of antibody for immunoprecipitation at $4{ }^{\circ} \mathrm{C}$ for $3 \mathrm{~h}$. The Sepharose beads were intensively washed with lysis buffer containing $0.5 \mathrm{M} \mathrm{NaCl}$. The precipitates were then subjected to SDS-PAGE and immunoblot analysis.

\section{RNA interference (RNAi) constructs}

The human IFITM3-RNAi constructs were generated using the pSUPER-retro vector (OligoEngine, Seattle, WA, USA) according to the manufacturer's protocol. The target sequences for the human IFITM3 constructs were as follows: \#1, 5'-TGCTGA TCTTCCAGGCCTA-3' and \#2, 5'-TCGTCTGGTCCCTGTT CAA- $3^{\prime}$. An RNAi construct targeting GFP was used as the control RNAi.

\section{Quantitative real-time PCR (qPCR)}

Total RNA was extracted from cells with TRIzol Reagent and subjected to real-time PCR analysis. The mRNA levels of specific genes were normalized to that of glyceraldehyde 3-phosphate dehydrogenase (GAPDH) mRNA. The genespecific primer sequences were as follows: GAPDH, 5'-GAGT CAACGGATTTGGTCGT-3' (forward), 5'-GACAAGCTTCC CGTTCTCAG-3' (reverse); IFNB1, 5'-TTGTTGAGAACCTCC TGGCT-3' (forward), 5'-TGACTATGGTCCAGGCACAG-3' (reverse); RANTES, 5'-GGCAGCCCTCGCTGTCATCC-3' (forward), 5'-GCAGCAGGGTGTGGTGTCCG-3' (reverse); ISG56, 5'-TCATCAGGTCAAGGATAGTC-3' (forward), 5' - CC ACACTGTATTTGGTGTCTAGG-3' (reverse); IRF3, 5'-TCTG CCCTCAACCGCAAAGAAG-3' (forward), 5'-TACTGCCTC CACCATTGGTGTC-3' (reverse); IFITM3, 5'-CTGGGCTTCA TAGCATTCGCCT-3'(forward), 5'-AGATGTTCAGGCACTT GGCGGT-3'(reverse).

\section{ELISA}

The supernatants of the cell culture medium were analyzed using a human IFN- $\beta$ (PBL) ELISA kit following the protocols recommended by the manufacturers.

\section{In vitro binding assay}

For the glutathione S-transferase (GST) pull-down assay, GST fusion constructs were transformed into bacterial strain BL21. 
The fusion proteins were induced with $0.1 \mathrm{mM} \mathrm{IPTG}$ at $18^{\circ} \mathrm{C}$ overnight and purified with Glutathione-Sepharose 4B (GE healthcare) according to the manufacturer's instructions. The purified GST-tagged proteins were then incubated with the HEK293 cell extracts. The protein complex was pulled down with Glutathione-Sepharose 4B beads and then subjected to western blot analysis.

\section{Statistical analysis}

Data were statistically analyzed by the Student's $t$-test. $P$-values less than 0.05 were considered statistically significant.

\section{RESULTS}

Overexpression of IFITM3 inhibits SeV-triggered induction of IFN- $\beta$

IFITM3 blocks the infection of many viruses that require endosomal entry pathways. ${ }^{14}$ However, whether IFITM3 affects virus-induced production of IFN is still unknown. $\mathrm{SeV}$ is not inhibited by IFITM3 (Supplementary Figure S1a), possibly because it enters cells by fusing to the cell surface. ${ }^{18}$ Thus, we infected HEK293 cells with $\mathrm{SeV}$ and performed luciferase assays to identify the role of IFITM3 in RLR-mediated antiviral signaling. Overexpression of IFITM3 inhibited $\mathrm{SeV}$-induced activation of the IFN $\beta$ promoter, ISRE and NF- $\mathrm{KB}$ in a dosedependent manner in HEK293 cells (Figure 1a and Supplementary Figure S1b). Similar results were observed in HeLa cells (Supplementary Figure S1c), suggesting that the effects of IFITM3 on SeV-triggered antiviral signaling were not cell-type-specific. Overexpression of IFITM3 had no marked effect on IFN $\gamma$-triggered activation of the IRF1 reporter in either HEK293 or HeLa cells (Figure 1b and Supplementary Figure S1d) or TLR3 signaling (data not shown), suggesting that IFITM3 specifically inhibits virus-triggered activation of the RLR pathway. Although IFITM1 and IFITM2 have high sequence identities with IFITM3 (61\% and 90\%, respectively), they had no marked effect on SeV-induced activation of IFN $\beta$ and ISRE (Figure 1c). We extended the analysis by evaluating the transcription of IFNB1 and its downstream genes. The SeVtriggered or transfected-poly(I:C)-induced transcription of the IFNB1, RANTES and ISG56 genes was inhibited by overexpression of IFITM3 (Figures $1 \mathrm{~d}$ and e). $\mathrm{SeV}$ or poly(I:C)induced secretion of IFN $\beta$ into the medium was also impaired by overexpression of IFITM3 (Figures $1 \mathrm{f}$ and g). These results suggest that IFITM3 negatively regulates cellular antiviral signaling.

Knockdown of IFITM3 potentiates virus-triggered induction of IFN- $\beta$ and inhibits viral replication

To confirm the role of endogenous IFITM3 in RLR-mediated production of type I IFNs, we prepared two RNAi constructs and determined their effects on the knockdown of IFITM3. As shown in Figure 2a, \#2 IFITM3-RNAi transfection inhibited IFITM3 levels to $10 \%$ of the control sample $(P<0.01)$. The \#1 RNAi transfection inhibited IFITM3 levels to $60 \%$ of the control sample (Figure 2a). In the reporter assays, knockdown of IFITM3 by RNAi transfection significantly potentiated SeV- triggered activation of the IFN- $\beta$ promoter, ISRE and NF- $\mathrm{kB}$, but not IFN- $\gamma$-triggered activation of the IRF1 promoter (Figures 2b and c; Supplementary Figure S1e). The degree of potentiation correlated with the knockdown efficiency of the corresponding RNAi construct (Figure $2 \mathrm{~b}$ and Supplementary Figure S1e). Because the effect of the \#2 RNAi construct was better than that of the \#1 RNAi construct, we selected the IFITM3-RNAi\#2 construct for the experiments described below. Knockdown of IFITM3 by the RNAi construct (IFITM3i) potentiated $\mathrm{SeV}$ - and transfected-poly(I:C)-induced transcription of IFNB1, RANTES and ISG56 (Figures $2 \mathrm{~d}$ and e), as well as the secretion of IFN $\beta$ into the medium (Figures $2 \mathrm{f}$ and g).

Next, we evaluated the effect of IFITM3 on viral replication. The conditioned medium from cultured HEK293 cells, which had been transfected with the IFITM3i construct and poly(I:C), was collected and used to treat Vero cells as previously described. ${ }^{23}$ The treated Vero cells were then infected with GFP-VSV or GFP-NDV, and replication of the GFP-labeled viruses was evaluated by direct observation under microscopy. The green fluorescence representing VSV or NDV particles decreased markedly in cells treated with the conditional medium from IFITM3-knockdown cells compared with the control groups (Figure $2 \mathrm{~h}$ ), suggesting that IFITM3 plays inhibitory roles in virus-triggered induction of IFN- $\beta$ and subsequently inhibits viral replication.

\section{IFITM3 regulates virus-triggered signaling at the level of IRF3}

To determine the molecular order of IFITM3 in the virustriggered signaling pathway, we examined the effects of IFITM3 on the transcription of IFNB1 mediated by components of the virus-triggered pathway. As shown in Figure 3a, IFITM3 inhibited the transcription of IFNB1 induced by overexpression of RIG-I-CARD, VISA, TBK1 and IRF3-5D. Consistent with this finding, knockdown of IFITM3 by RNAi potentiated the transcription of IFNB1 mediated by overexpression of IRF3 (Figure 3b). Because activation of the transcription factor IRF3 is a critical event in antiviral signaling, we next determined whether the activation status of IRF3 was affected by IFITM3. $\mathrm{SeV}$-induced dimerization and nuclear translocation of IRF3 were increased in IFITM3-knockdown cells (Figure $3 \mathrm{c}$ and Supplementary Figure S2a). The total amount of IRF3 also increased dramatically when IFITM3 was knocked down (Figure 3c). To confirm this finding, we calculated the ratio of the amount of IRF3 dimer to the total amount of IRF3 by measuring the grayscale values of the bands in Figure 3c. The results showed that knockdown of IFITM3 enhanced the dimerization of IRF3 (Figure 3d). However, the transcription level of IRF3 remained unchanged when IFITM3 was knocked down, suggesting that IFITM3 did not affect IRF3 expression at the level of transcription (Figure 3e). To determine whether IFITM3 affected the protein levels of IRF3, we evaluated the expression of IRF3 after stimulation with $\mathrm{SeV}$. The protein levels of IRF3 greatly decreased when IFITM3 was overexpressed, whereas knockdown of IFITM3 enhanced the 
a
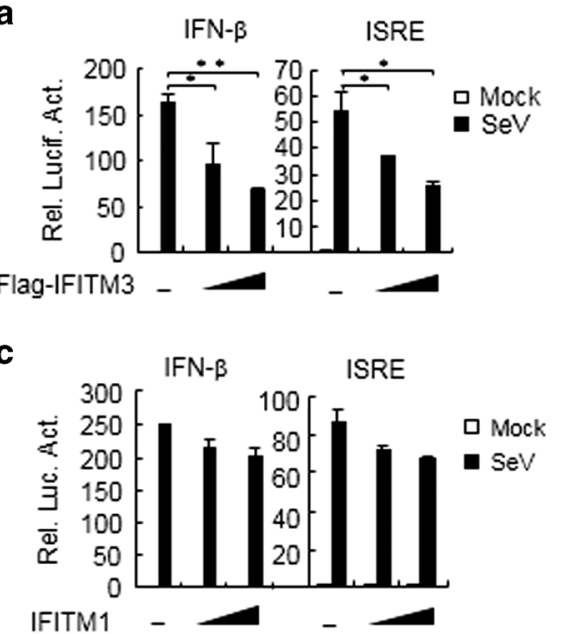

d

IFNB1 RANTES ISG56

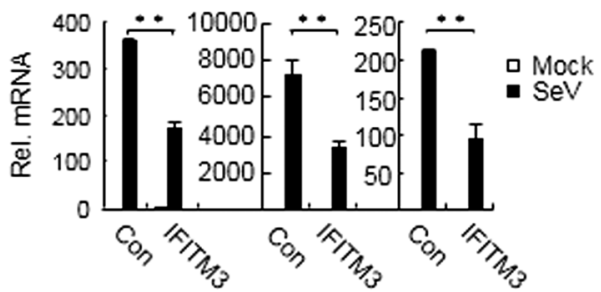

e

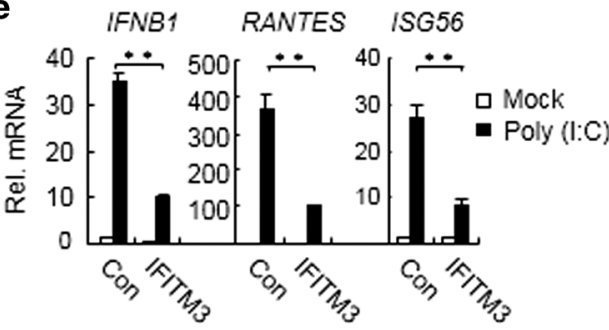

b
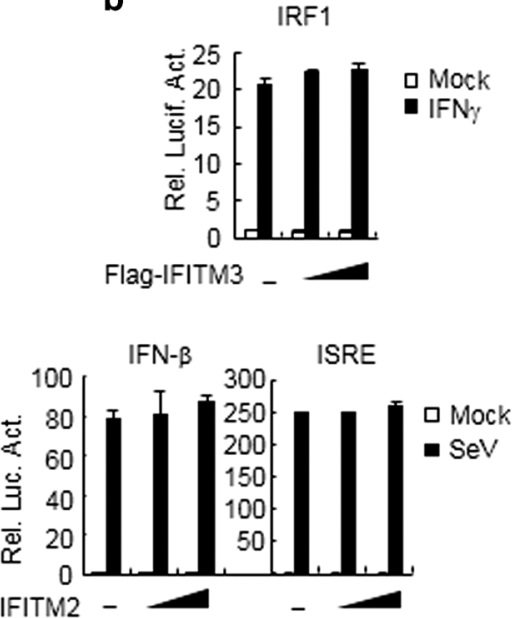

f

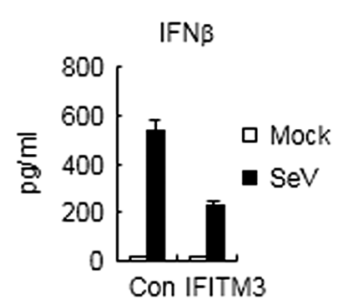

g

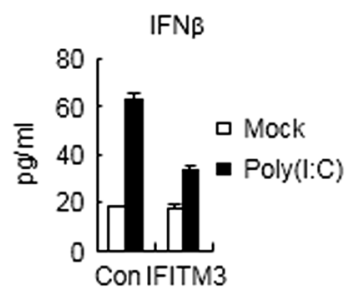

Figure 1 Overexpression of IFITM3 inhibits virus-triggered induction of IFN- $\beta$. (a and c) HEK293 cells were transfected with the indicated reporters and IFITM3 (a) or IFITM1/2 (c) expression plasmids $\left(0,25,50 \mathrm{ng}\right.$ per $5 \times 10^{5}$ cells). Twenty-four hours after transfection, the cells were infected with SeV or left untreated for $12 \mathrm{~h}$ before luciferase assays were performed. (b) HEK293 cells were transfected with the IRF1 promoter reporter and the indicated plasmids. The cells were treated with IFN- $\gamma$ or left untreated before performing the luciferase assays. $(\mathbf{d}-\mathbf{g})$ HEK293 cells were transfected with control or Flag-IFITM3 (100 ng per $10^{6}$ cells) plasmid for $24 \mathrm{~h}$. The cells were infected with $\mathrm{SeV}$ (d and $\mathbf{f}$ ) for $10 \mathrm{~h}$ or transfected with poly(I:C) (e and $\mathbf{g}$ ) followed by real-time PCR (d and e) or ELISA analysis (f and $\mathbf{g})$. (a-f) Graphs show the means \pm s.d., $n=3,{ }^{*} P<0.05, * * P<0.01$.

IRF3 levels even without $\mathrm{SeV}$ infection (Figures $3 \mathrm{f}$ and $\mathrm{g}$ ). Consistently, phosphorylation of IRF3 dramatically decreased when IFITM3 was overexpressed and increased when IFITM3 was knocked down (Supplementary Figure S2b). In contrast, the expression levels of IFITM3 had no marked effect on the other proteins in this pathway, such as TBK1 or TBK phosphorylation (Figures $3 \mathrm{f}$ and $\mathrm{g}$ and Supplementary Figure S2b). IFITM1 or IFITM2 had no marked effect on the basal level of IRF3 (Figure 3h). Collectively, these data suggest that IFITM3 regulates antiviral signaling at the level of IRF3 and participates in the regulation of the basal expression of IRF3.

\section{IFITM3 associates with IRF3}

To explore how IFITM3 regulates the expression of IRF3, we performed coimmunoprecipitation to determine whether
IFITM3 interacted with IRF3. As shown in Figure 4a, IFITM3 interacted constitutively with endogenous IRF3, but not VISA, with or without $\mathrm{SeV}$ stimulation. Furthermore, IRF3-5A, which is the phosphorylation-deficient mutant of IRF3, was also found to interact with IFITM3 (Figure 4b), suggesting that the interaction between IFITM3 and IRF3 did not rely on the phosphorylation of IRF3. To further confirm this interaction, we performed an in vitro binding assay. Bacterially expressed and affinity-purified GST-IFITM3 fusion protein was incubated with ectopic IRF3 or IRF3-5A-expressing whole-cell extracts. As shown in Figure 4c, both IRF3 and IRF3-5A were pulled down by GST-IFITM3, suggesting that the interaction between IFITM3 and IRF3 was constitutive and independent of the activation status of IRF3. Consistent with this finding, a confocal immunofluorescence analysis showed that IFITM3 
a

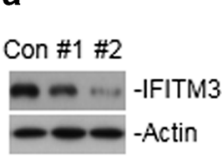

b

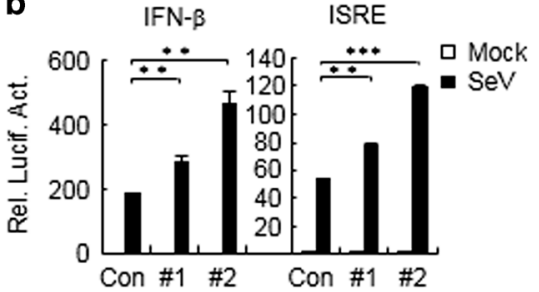

C

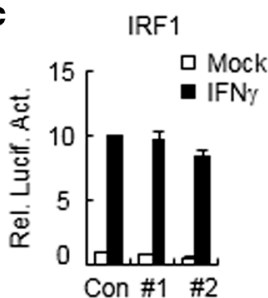

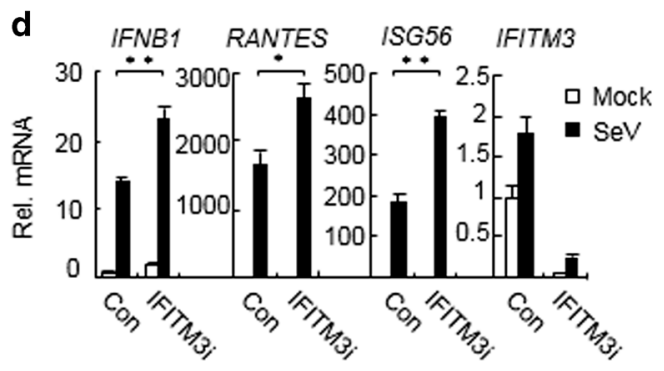

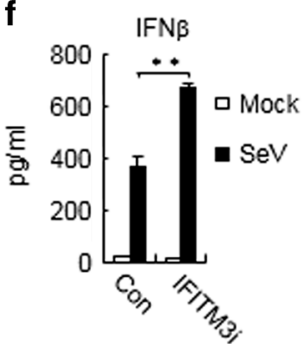

e

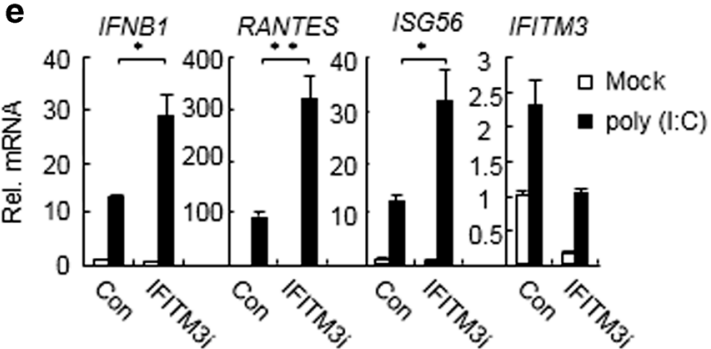

h
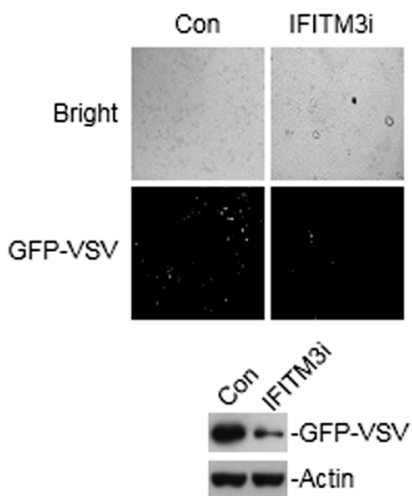
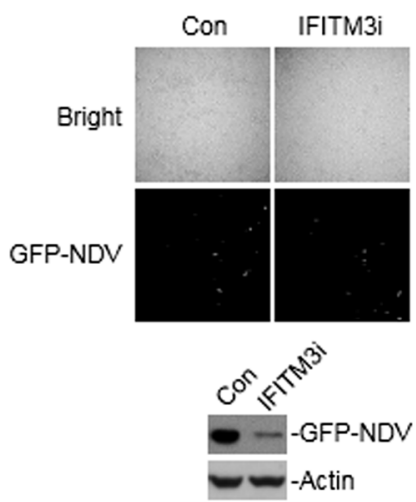

Figure 2 Knockdown of IFITM3 potentiates virus-triggered induction of IFN- $\beta$ and inhibits viral replication. (a) HeLa cells were transfected with the control or indicated RNAi constructs. Cell lysates were analyzed on immunoblots with the indicated antibodies. Graphs show the means \pm s.d., $n=3,{ }^{*} P<0.05,{ }^{*} P<0.01$. (b and $\mathbf{c}$ ) HEK293 cells were transfected with the indicated RNAi constructs together with the indicated reporter plasmids. The cells were then infected with SeV (b) or IFN- $\gamma$ (c) for $12 \mathrm{~h}$ before luciferase assays were performed. (d-g) HEK293 cells were transfected with the indicated RNAi constructs. Cells were then infected with SeV (d and f) for $10 \mathrm{~h}$ or transfected with poly(I:C) (e and $\mathbf{g}$ ) before real-time PCR (d and e) or ELISA ( $\mathbf{f}$ and $\mathbf{g}$ ) was performed. (h) HEK293 cells were transfected with control or IFITM3-RNAi construct for $36 \mathrm{~h}$ and then transfected with poly(I:C) for $24 \mathrm{~h}$. The supernatants were collected and applied to Vero cells for $24 \mathrm{~h}$. These Vero cells were infected with GFP-VSV or GFP-NDV $(\mathrm{MOI}=0.1)$ for $24 \mathrm{~h}$ and visualized by microscopy or analyzed on immunoblots with the indicated antibodies.

co-localized with IRF3 in the cytoplasm of HeLa cells (Supplementary Figure S2c). Domain mapping analysis indicated that the $\mathrm{N}$ terminus of IRF3 (amino acids 1-140) was responsible for its interaction with IFITM3 (Figure 4d). Because IFITM3 was induced by IFNs, we analyzed the kinetic pattern of IFITM3 and IRF3. The expression of IFITM3 was induced while the expression of IRF3 was decreased following
IFN $\alpha$ or poly(I:C) transfection treatment (Figure 4e). These data suggest that IFITM3 associates with IRF3 and possibly mediates the degradation of IRF3.

IFITM3 mediates autophagic degradation of IRF3

IRF3 degradation is mediated by proteasomal degradation and/ or autophagy. ${ }^{24-26}$ To investigate the role of IFITM3 in IRF3 
a

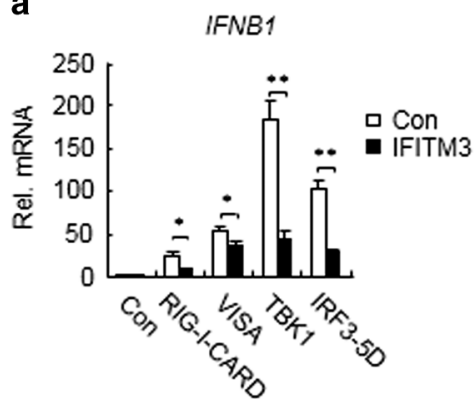

b

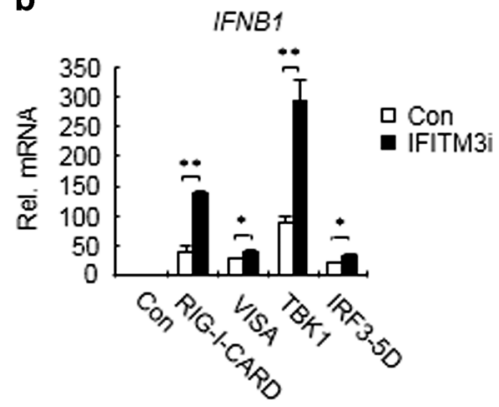

C
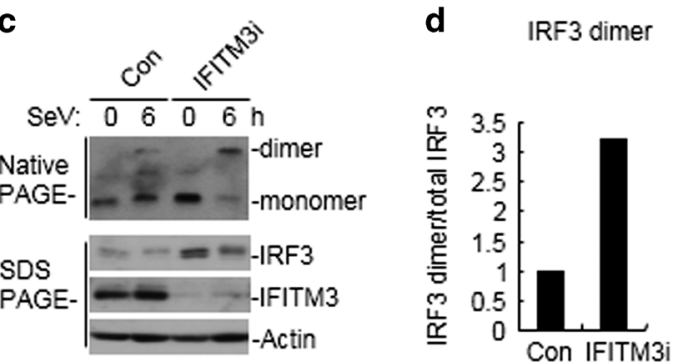

f
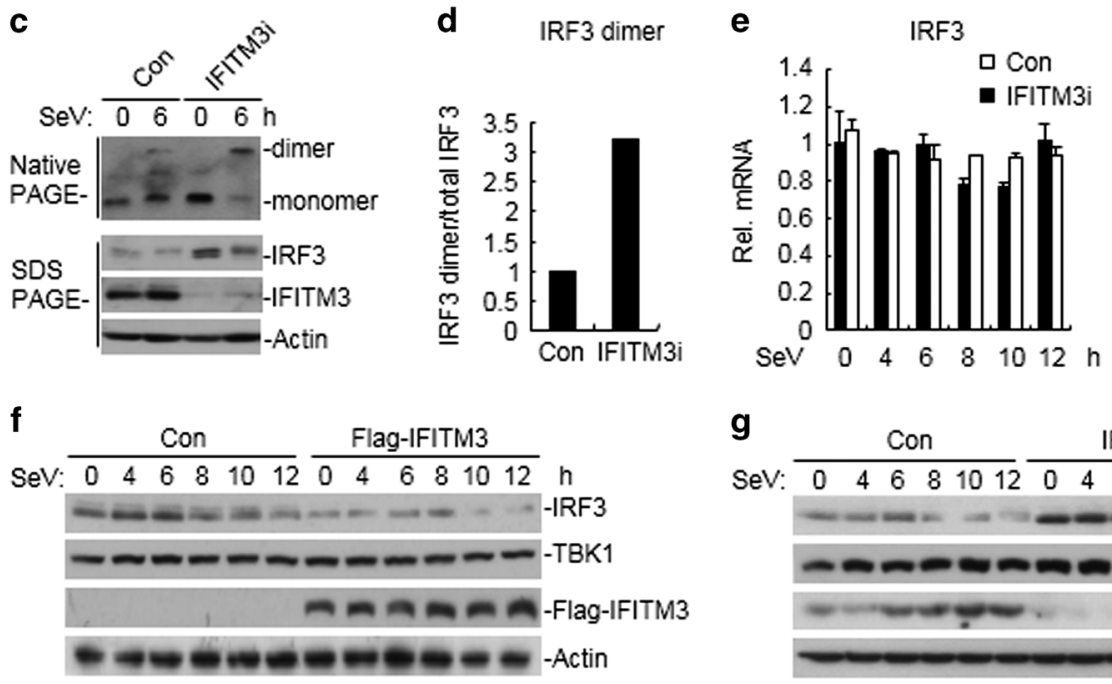

g
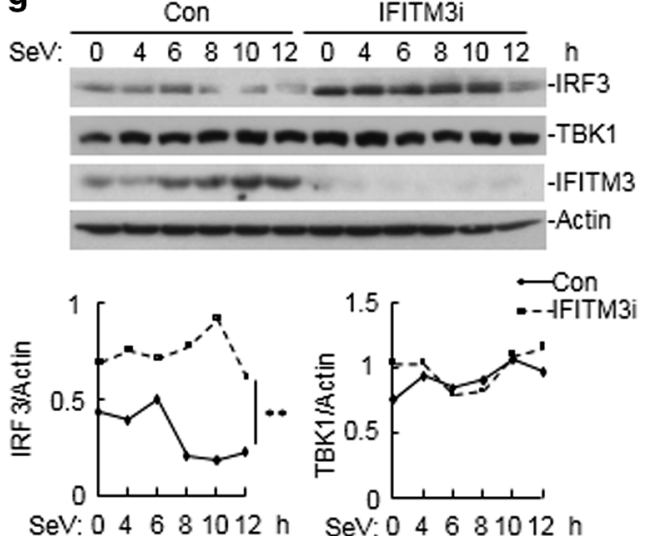
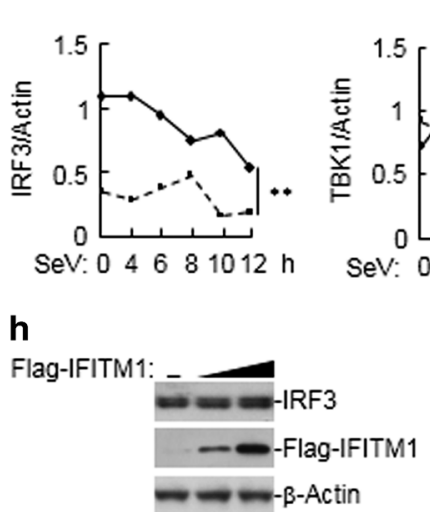

$\_$Con

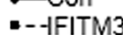

h

Flag-IFITM1:

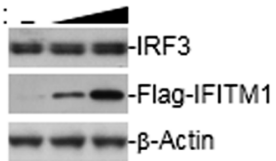

Flag-IFITM2

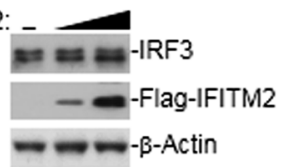

Figure 3 IFITM3 regulates virus-triggered signaling at the level of IRF3. (a) HEK293 cells were transfected with the indicated expression plasmids together with control or IFITM3 plasmids for $24 \mathrm{~h}$. Real-time PCR was performed $24 \mathrm{~h}$ later. (b) Stable IFITM3-knockdown HEK293 cells were transfected with the indicated expression plasmids and then analyzed by real-time PCR. Graphs show means \pm s.d., $n=3$, ${ }^{*} P<0.05,{ }^{*} P<0.01$. (c and d) Stable IFITM3-knockdown HEK293 cells were infected with SeV or left uninfected for $8 \mathrm{~h}$. The cell lysates were separated by native (top) or SDS (bottom) PAGE and analyzed on immunoblots with the indicated antibodies (c). The ratio of the IRF3 dimer to total IRF3 was calculated by measuring the grayscale values of the bands (d). (e) IRF3 mRNA levels in stable IFITM3knockdown HEK293 cells after SeV stimulation were measured by real-time PCR. (f and g) HEK293 cells were transfected with IFITM3 plasmid (f) or IFITM3-RNAi construct $(\mathbf{g})$. The cells were then infected with SeV and analyzed on immunoblots with the indicated antibodies. The ratio of IRF3 or TBK1 to actin was calculated by measuring the grayscale values of the bands and is shown in the lower panels. (h) HEK293 cells were transfected with increased amounts of IFITM1 or IFITM2 plasmids. The endogenous level of IRF3 was then detected by immunoblotting.

degradation, we prepared an RNAi-off-target IFITM3 mutant (Flag-IFITM3-M) with three nucleotide nonsense mutations in the target sequence of the IFITM3-RNAi construct. As shown in Supplementary Figure S3a, the IFITM3-RNAi construct could inhibit the expression of Flag-IFITM3 but not FlagIFITM3-M. Next, we reconstituted the expression of IFITM3 in stable IFITM3-knockdown 293 cells by overexpressing Flag-IFITM3-M, and treated the cells with the proteasome 
a

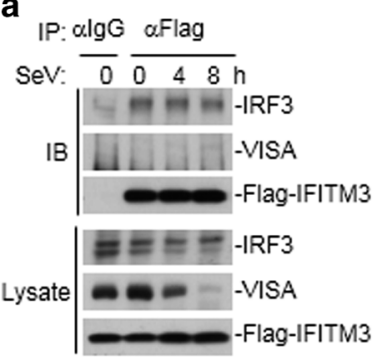

b

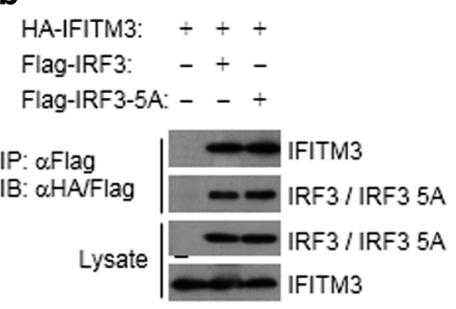

c

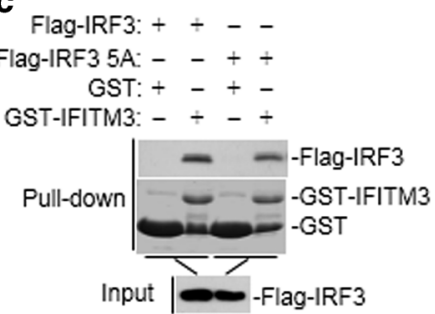

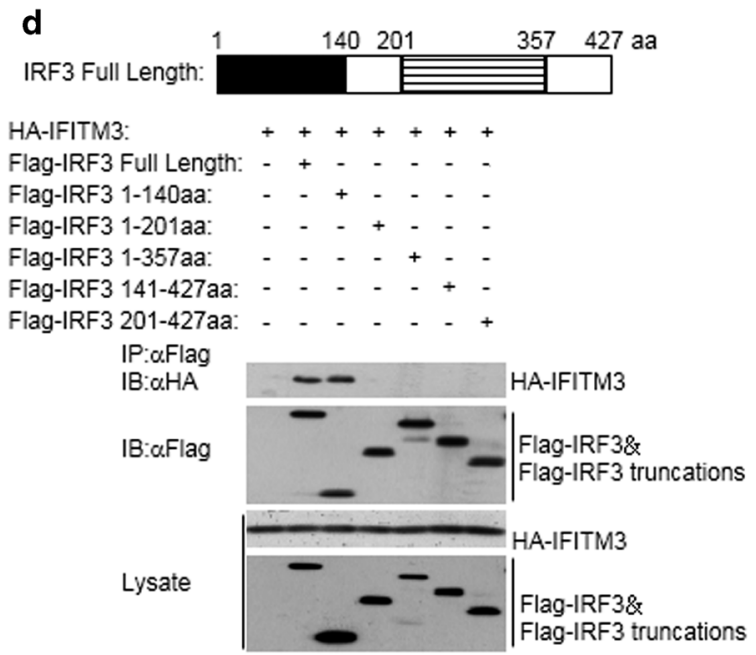

e

IFN $\alpha: 0 \begin{array}{lllll}0 & 4 & 8 & 12 & h\end{array}$

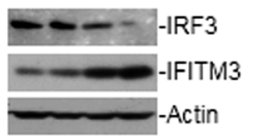

Poly(l:C): $01224 \mathrm{~h}$

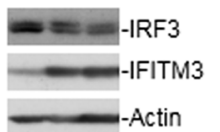

Figure 4 IFITM3 Associates with IRF3. (a) HEK293 cells were transfected with Flag-IFITM3 plasmid and infected with SeV. The cell lysates were tested by coimmunoprecipitation and analyzed on immunoblots with the indicated antibodies. (b) HEK293 cells were transfected with HA-IFITM3 and Flag-IRF3 or Flag-IRF3-5A plasmid. The cell lysates were coimmunoprecipitated and analyzed by immunoblots with the indicated antibodies. (c) Glutathione-Sepharose beads coupled to GST-IFITM3 were incubated with HEK293 cell extracts expressing Flag-IRF3 or Flag-IRF3-5A. Proteins bound to the beads were analyzed by western blotting. The experiment was repeated twice with comparable results. (d) Mapping of the minimal interaction domains between IRF3 and IFITM3. HEK293 cells $\left(1 \times 10^{7}\right)$ were transfected with the indicated plasmids. Coimmunoprecipitation and immunoblots were performed. (e) HeLa cells were treated with IFN $\alpha$ (upper panel) or transfected with poly(I:C) (lower panel) for the indicated times. Cell lysates were then applied to the immunoblot.

inhibitor MG132 or the autophagy inhibitor 3-MA. MG132 treatment had little effect on the degradation of IRF3 caused by overexpression of Flag-IFITM3-M (Figure 5a). However, 3-MA treatment could inhibit the degradation of IRF3 caused by overexpression of Flag-IFITM3-M (Figure 5a), suggesting that the IFITM3-mediated degradation of IRF3 was autophagydependent but not proteasome-dependent. To confirm this finding, we first assessed whether IRF3 was degraded through autophagy in our system. Silencing autophagy-related 7 (ATG7), which is a key factor during the synthesis of the autophagosome precursor, leads to the inhibition of autophagy. ${ }^{27}$ Mouse atg7-deficient ( $\operatorname{atg} 7^{-/-}$) MEF cells and control wild-type (WT) cells were treated with cycloheximide $(\mathrm{CHX})$, an inhibitor of new protein synthesis. Immunoblotting analysis showed that the level of IRF3 was higher in $\operatorname{Atg} 7^{-/-}$ cells than in WT cells after treatment with CHX (Supplementary Figure S3b), confirming previous reports that autophagy is one of the turnover mechanisms of IRF3. ${ }^{26}$ It has been reported that overexpression of IFITM3 can induce autophagy, including LC3 puncta and lipidation. ${ }^{28,29}$ Consistent with this finding, IFITM3 dose-dependently promoted the transformation of type I LC3 to type II LC3, which is an indicator of enhanced autophagy (Figure 5b). A confocal immunofluorescence analysis showed that IFITM3 colocalized with autophagic components such as LC3, ATG5 and Beclin1 (Figures $5 \mathrm{c}$ and $\mathrm{d}$ ). Moreover, IFITM3 or IRF3 interacted with LC3 and Beclin1 (Figure 5e), suggesting that LC3 and Beclin1 are involved in the process of IFITM3 mediating the autophagic degradation of IRF3. Overexpression of IFITM3 changed the subcellular location of IRF3 from a disperse distribution in the cytosol to specific organelle autophagosomes, as indicated by LC3-GFP (Figure 5f), suggesting that IFITM3 mediates the autophagic degradation of IRF3. Furthermore, viral infection induced the majority of IRF3 to translocate to the nucleus, but a portion of IRF3 colocalized with LC3, suggesting that $\mathrm{SeV}$ could indeed induce the translocation of cytosolic IRF3 into autophagosomes (Figure 5f). In HEK293 cells treated with CHX, IRF3 was degraded much more slowly when IFITM3 was silenced in comparison to the control cells (Figure $5 \mathrm{~g}$ ), suggesting that IFITM3-mediated autophagic degradation of IRF3 was critical for the turnover of IRF3. 
a

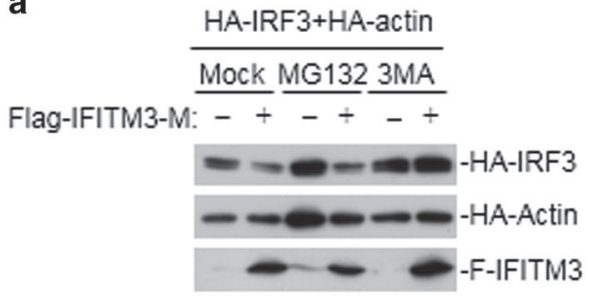

b

Flag-IFITM3:

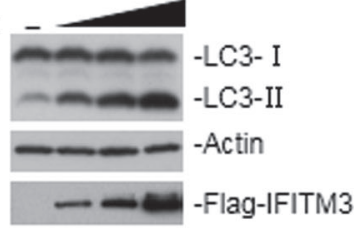

e

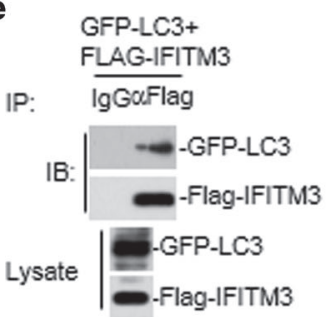

$$
\begin{array}{ll} 
& \text { GFP-LC3+ } \\
\text { IP: } & \frac{\text { FLAG-IRF3 }}{\lg \text { Goflag }}
\end{array}
$$

IB:

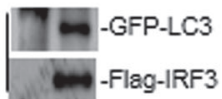

Lysate

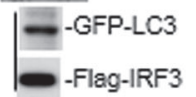

C

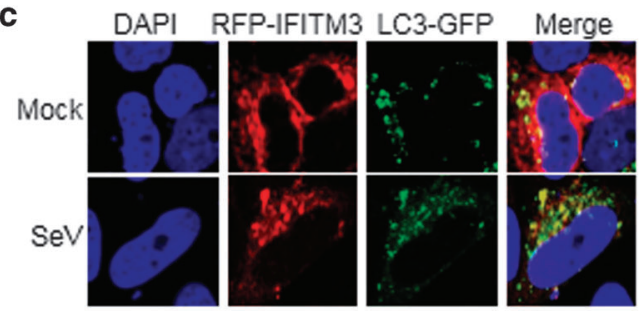

d

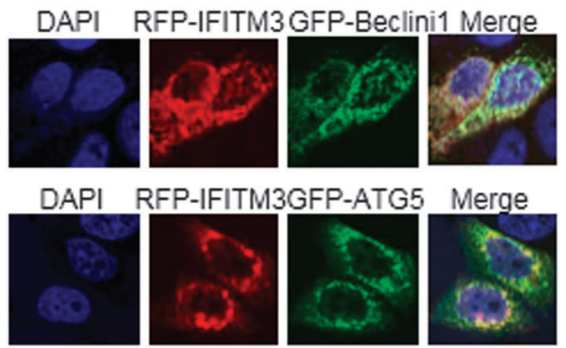

f
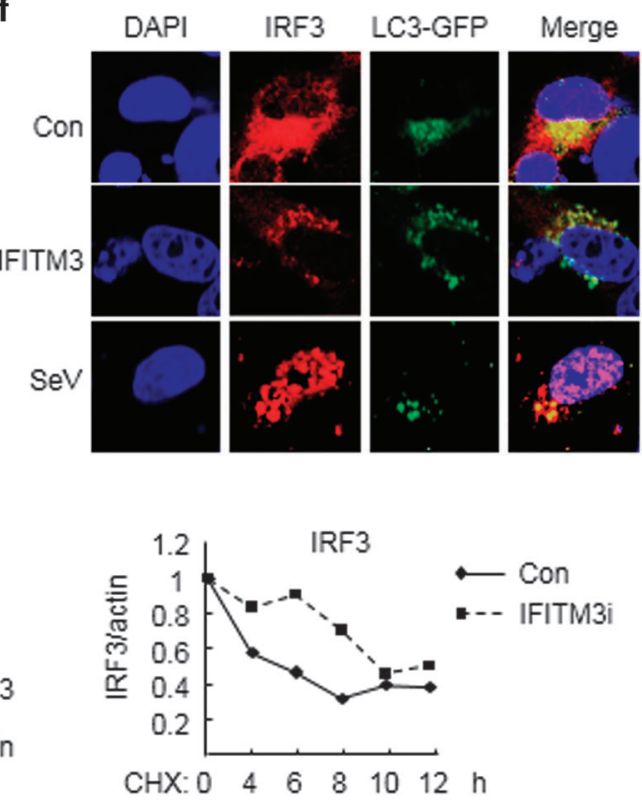

Figure 5 IFITM3 mediates autophagic degradation of IRF3. (a) Stable IFITM3-knockdown HEK293 cells were transfected with the indicated plasmids and treated with 3-MA or MG132 for $6 \mathrm{~h}$ before an immunoblot analysis was performed. (b) HEK293 cells were transfected with increasing amounts of Flag-IFITM3 and analyzed on immunoblots with the indicated antibodies. (c and d) HeLa cells transfected with the indicated plasmids were infected with SeV for $12 \mathrm{~h}$ or left untreated and then analyzed under immunofluorescence microscopy. (e) HEK293 cells were transfected with the indicated plasmids and applied to the coimmunoprecipitation and immunoblot experiments. (f) HeLa cells were transfected with Flag-IFITM3 and LC3-GFP plasmids, or infected with SeV. Immunofluorescence assays were performed using anti-IRF3 antibody as the primary antibody. (g) Stable IFITM3-knockdown HEK293 cells were infected with SeV and treated with $\mathrm{CHX}$ for the indicated times before immunoblot analysis was performed (left panel). The expression levels of IRF3 and actin were semi-quantified by measuring the grayscales of the bands on the western blots. The normalized expression of IRF3 is shown in the line chart (right panel).

\section{DISCUSSION}

IFITM3 has been extensively studied as a restriction factor that confers broad resistance to viral infection. ${ }^{13,14}$ The enveloped and nonenveloped viruses that are restricted by IFITM3 utilize an endosomal entry mechanism. ${ }^{30,31}$ IFITM3 restricts their replication by blocking virus-endosome fusion. ${ }^{29,32}$ A possible underlying mechanism is that IFITM3 induces the accumulation of cholesterol in multi-vesicular bodies and in late endosomes, thus impairing the membrane fusion of intraluminal virion-containing vesicles and endosomes. ${ }^{33}$ IFITM3 is also strongly retained in resident memory $\mathrm{CD}^{+} \mathrm{T}$ cells to facilitate cell survival and enhance cell resistance to infection with influenza viruses. ${ }^{34}$

Here we report a novel role of IFITM3 in regulating the virus-induced production of type I IFNs. Using $\mathrm{SeV}$ or the 
dsRNA analog poly(I:C) as the agonist, we have shown that overexpression of IFITM3, but not IFITM1 or IFITM2, inhibits cytosolic-RNA-induced activation of the IFN- $\beta$ promoter and the transcription of IFNB1 and its downstream antiviral genes, whereas the knockdown of IFITM3 has the opposite effects. Knockdown of IFITM3 enhances the dimerization and nuclear translocation of IRF3, and it promotes the antiviral signaling pathway against viral replication. These results suggest that IFITM3, the expression of which is enhanced by viral infection and type I IFNs, regulates the virus-triggered antiviral response in a negative-feedback manner. Many ISGs not only block viral replication during different phases of the viral replication cycle, but they also regulate the production of IFNs to maintain suitable antiviral responses. For example, ISG56 negatively regulates cellular antiviral responses by disrupting the interactions between mediator of IRF3 activation and VISA or TBK1. ${ }^{35}$ Type I IFN leads to the induction of RBCK1, which can induce the subsequent degradation of IRF3. ${ }^{36}$ PCBP2, another ISG, acts as a scaffold to facilitate AIP4-mediated degradation of VISA, which is a critical mechanism in the negative regulation of RLR signaling and is also apparent in Figure $4 a^{37}$ By contrast, DDX60 enhances RLR-mediated production of IFNs by binding to RIG-I and promoting the binding of RIG-I to dsRNA. ISG15 regulates type I IFN signaling by modulating the ISGylation of RIG-I and IRF3. ${ }^{38-40}$ Our study adds IFITM3 to the ISGs that negatively regulate virus-induced induction of type I IFNs while simultaneously inhibiting viral replication. As is well known, immoderate immune responses lead to pathological tissue damage and even induce autoimmune reactions. The inhibitory effects of IFITM3 on both IFN production and viral replication are undoubtedly important for maintaining a suitable level of innate antiviral responses.

The role of IFITM3 in restricting viruses is considered to be closely related to its cellular localization. IFITM3 partially localizes to acidic compartments, including late endosomes expressing Rab5 and Rab7, lysosomes expressing LAMP1, and autolysosomes expressing LC3 and CD63. ${ }^{28,29}$ IFITM3 restriction of SARS-CoV is circumvented when trypsin digestion is used to trigger membrane fusion at or near the plasma membrane rather than within the acidic cellular compartment. ${ }^{32}$ In this study, we found that the role of IFITM3 in regulating IFN signaling is also closely related to its cellular localization. We observed that overexpression of IFITM3 led to an increase in LC3 transformation and the formation of LC3 puncta (Figures $5 b$ and $\mathrm{f}$ ). Based on the substantial increase in IRF3 when IFITM3 is depleted (Figure 3c), we demonstrated that IRF3 and IFITM3 interact constitutively with one another and colocalize with the same cellular autophagosome compartments (Figures $4 \mathrm{a}-\mathrm{d}$ and Supplementary Figure S2c). Because the autophagy inhibitor 3-MA rescued the degradation of IRF3 caused by IFITM3 (Figure 5a), we confirmed that autophagy is one mechanism of IRF3 homeostasis and that overexpression of IFITM3 enhances the process of autophagy, as evidenced by the transformation of LC3 from type I to type II. Finally, but importantly, we have provided evidence that IRF3 is concentrated in autolysosomes expressing LC3 when IFITM3 is overexpressed (Figure 5f). Upon stimulation by viruses, high levels of IFITM3, induced by IFNs, enhance the degradation of IRF3 by autophagy and thus regulate antiviral immune responses via a negativefeedback mechanism. Although in this study we have shown that IFITM3 negatively regulates the activation of NF- $\mathrm{NB}$ (Supplementary Figures S1b and S1e), it is still unclear whether this function is related to autophagy, necessitating further study.

In summary, our study reveals a novel function of IFITM3 in antiviral immunity. IFITM3 not only restricts many viruses but also negatively regulates type I IFN signaling by enhancing the autophagic degradation of IRF3. Our findings demonstrate a previously undescribed role of IFITM3 in regulating cellular antiviral responses.

\section{CONFLICT OF INTEREST}

The authors declare no conflict of interest.

\section{ACKNOWLEDGEMENTS}

We thank Dr Chen Liang, Dr Wentao Qiao and Dr Rongjia Zhou for providing plasmids and stimulating discussion. This work was supported by grants from the Ministry of Science and Technology of China (2014CB910103), the National Natural Science Foundation of China $(31370867,31521091)$ and the Fundamental Research Funds for the Central University (2042015kf0182). LQJ, HBS and YL designed the research; LQJ, TX, YHH, SY, MSS, CQL and GJH performed the research; LQJ and YL analyzed the data and wrote the paper.

1 Akira S, Uematsu S, Takeuchi O. Pathogen recognition and innate immunity. Cell 2006; 124: 783-801.

2 O'Neill LA, Bowie AG. Sensing and signaling in antiviral innate immunity. Curr Biol 2010; 20: R328-R333.

3 Beutler B, Eidenschenk C, Crozat K, Imler JL, Takeuchi O, Hoffmann JA et al. Genetic analysis of resistance to viral infection. Nat Rev Immunol 2007; 7: 753-766.

4 Andrejeva J, Childs KS, Young DF, Carlos TS, Stock N, Goodbourn S et al. The $\mathrm{V}$ proteins of paramyxoviruses bind the IFN-inducible RNA helicase, mda-5, and inhibit its activation of the IFN-beta promoter. Proc Natl Acad Sci USA 2004; 101: 17264-17269.

5 Xu LG, Wang YY, Han KJ, Li LY, Zhai Z, Shu HB. VISA is an adapter protein required for virus-triggered IFN-beta signaling. Mol Cell 2005; 19: 727-740.

6 Seth RB, Sun L, Ea CK, Chen ZJ. Identification and characterization of MAVS, a mitochondrial antiviral signaling protein that activates NF-kappaB and IRF 3. Cell 2005; 122: 669-682.

7 Kawai T, Takahashi K, Sato S, Coban C, Kumar H, Kato H et al. IPS-1, an adaptor triggering RIG-I- and Mda5-mediated type I interferon induction. Nat Immunol 2005; 6: 981-988.

8 Meylan E, Curran J, Hofmann K, Moradpour D, Binder M, Bartenschlager $\mathrm{R}$ et al. Cardif is an adaptor protein in the RIG-I antiviral pathway and is targeted by hepatitis C virus. Nature 2005; 437: $1167-1172$.

9 Kawai T, Akira S. Toll-like receptors and their crosstalk with other innate receptors in infection and immunity. Immunity 2011; 34: 637-650.

10 Fitzgerald KA, McWhirter SM, Faia KL, Rowe DC, Latz E, Golenbock DT et al. IKKepsilon and TBK1 are essential components of the IRF3 signaling pathway. Nat Immunol 2003; 4: 491-496. 
11 Friedman RL, Manly SP, McMahon M, Kerr IM, Stark GR. Transcriptional and posttranscriptional regulation of interferon-induced gene expression in human cells. Cell 1984; 38: 745-755.

12 Reid LE, Brasnett AH, Gilbert CS, Porter AC, Gewert DR, Stark GR et al. A single DNA response element can confer inducibility by both alpha- and gamma-interferons. Proc Natl Acad Sci USA 1989; 86: 840-844.

13 Brass AL, Huang IC, Benita Y, John SP, Krishnan MN, Feeley EM et al. The IFITM proteins mediate cellular resistance to influenza A H1N1 virus, West Nile virus, and dengue virus. Cell 2009; 139: 1243-1254.

14 Anafu $\mathrm{AA}$, Bowen $\mathrm{CH}$, Chin $\mathrm{CR}$, Brass $\mathrm{AL}$, Holm GH. Interferoninducible transmembrane protein 3 (IFITM3) restricts reovirus cell entry. J Biol Chem 2013; 288: 17261-17271.

15 Jiang D, Weidner JM, Qing M, Pan XB, Guo H, Xu C et al. Identification of five interferon-induced cellular proteins that inhibit west nile virus and dengue virus infections. J Virol 2010; 84: 8332-8341.

16 Li K, Markosyan RM, Zheng YM, Golfetto O, Bungart B, Li M et al. IFITM proteins restrict viral membrane hemifusion. PLoS Pathog 2013; 9: e1003124

17 Yu J, Li M, Wilkins J, Ding S, Swartz TH, Esposito AM et al. IFITM proteins restrict HIV-1 infection by antagonizing the envelope glycoprotein. Cell Rep 2015; 13: 145-156.

18 Hach JC, McMichael T, Chesarino NM, Yount JS. Palmitoylation on conserved and nonconserved cysteines of murine IFITM1 regulates its stability and anti-influenza A virus activity. J Virol 2013; 87: 9923-9927.

19 Zhong B, Yang Y, Li S, Wang YY, Li Y, Diao F et al. The adaptor protein MITA links virus-sensing receptors to IRF3 transcription factor activation. Immunity 2008; 29: 538-550.

20 Lei CQ, Zhong B, Zhang Y, Zhang J, Wang S, Shu HB. Glycogen synthase kinase 3beta regulates IRF3 transcription factor-mediated antiviral response via activation of the kinase TBK1. Immunity 2010; 33: 878-889.

$21 \mathrm{Li} \mathrm{Y,} \mathrm{Chen} \mathrm{R,} \mathrm{Zhou} \mathrm{Q,} \mathrm{Xu} \mathrm{Z,} \mathrm{Li} \mathrm{C,} \mathrm{Wang} \mathrm{S} \mathrm{et} \mathrm{al.} \mathrm{LSm14A} \mathrm{is} \mathrm{a}$ processing body-associated sensor of viral nucleic acids that initiates cellular antiviral response in the early phase of viral infection. Proc Natl Acad Sci USA 2012; 109: 11770-11775.

22 Zhang J, Hu MM, Shu HB, Li S. Death-associated protein kinase 1 is an IRF3/7-interacting protein that is involved in the cellular antiviral immune response. Cell Mol Immunol 2014; 11: 245-252.

23 Versteeg GA, Rajsbaum R, Sanchez-Aparicio MT, Maestre AM, Valdiviezo J, Shi M et al. The E3-ligase TRIM family of proteins regulates signaling pathways triggered by innate immune pattern-recognition receptors. Immunity 2013; 38: 384-398.

24 Higgs R, Ni Gabhann J, Ben Larbi N, Breen EP, Fitzgerald KA, Jefferies CA. The E3 ubiquitin ligase Ro52 negatively regulates IFNbeta production post-pathogen recognition by polyubiquitin-mediated degradation of IRF3. J Immunol 2008; 181: 1780-1786.

25 Saitoh T, Tun-Kyi A, Ryo A, Yamamoto M, Finn G, Fujita T et al. Negative regulation of interferon-regulatory factor 3-dependent innate antiviral response by the prolyl isomerase Pin1. Nat Immunol 2006; 7: 598-605.
26 Kimura T, Jain A, Choi SW, Mandell MA, Johansen T, Deretic V. TRIMdirected selective autophagy regulates immune activation. Autophagy 2016; e-pub ahead of Print 16 March 2016; doi:10.1080/ 15548627.2016.1154254.

27 Komatsu M, Waguri S, Ueno T, Iwata J, Murata S, Tanida I et al. Impairment of starvation-induced and constitutive autophagy in Atg7deficient mice. J Cell Biol 2005; 169: 425-434.

28 Yount JS, Karssemeijer RA, Hang HC. S-palmitoylation and ubiquitination differentially regulate interferon-induced transmembrane protein 3 (IFITM3)-mediated resistance to influenza virus. J Biol Chem 2012; 287: 19631-19641.

29 Feeley EM, Sims JS, John SP, Chin CR, Pertel T, Chen LM et al. IFITM3 inhibits influenza A virus infection by preventing cytosolic entry. PLoS Pathog 2011; 7: e1002337.

30 Perreira JM, Chin CR, Feeley EM, Brass AL. IFITMs restrict the replication of multiple pathogenic viruses. J Mol Biol 2013; 425: 4937-4955.

31 Diamond MS, Farzan M. The broad-spectrum antiviral functions of IFIT and IFITM proteins. Nat Rev Immunol 2013; 13: 46-57.

32 Huang IC, Bailey CC, Weyer JL, Radoshitzky SR, Becker MM, Chiang JJ et al. Distinct patterns of IFITM-mediated restriction of filoviruses, SARS coronavirus, and influenza A virus. PLoS Pathog 2011; 7: e1001258.

33 Amini-Bavil-Olyaee S, Choi YJ, Lee JH, Shi M, Huang IC, Farzan M et al. The antiviral effector IFITM3 disrupts intracellular cholesterol homeostasis to block viral entry. Cell Host Microbe 2013; 13: 452-464.

34 Wakim LM, Gupta N, Mintern JD, Villadangos JA. Enhanced survival of lung tissue-resident memory CD8(+) T cells during infection with influenza virus due to selective expression of IFITM3. Nat Immunol 2013; 14: 238-245.

$35 \mathrm{Li} \mathrm{Y,} \mathrm{Li} \mathrm{C,} \mathrm{Xue} \mathrm{P,} \mathrm{Zhong} \mathrm{B,} \mathrm{Mao} \mathrm{AP,} \mathrm{Ran} \mathrm{Y} \mathrm{et} \mathrm{al.} \mathrm{ISG56} \mathrm{is} \mathrm{a}$ negative-feedback regulator of virus-triggered signaling and cellular antiviral response. Proc Natl Acad Sci USA 2009; 106: 7945-7950.

36 Zhang M, Tian Y, Wang RP, Gao D, Zhang Y, Diao FC et al. Negative feedback regulation of cellular antiviral signaling by RBCK1-mediated degradation of IRF3. Cell Res 2008; 18: 1096-1104.

37 You F, Sun H, Zhou X, Sun W, Liang S, Zhai Z et al. PCBP2 mediates degradation of the adaptor MAVS via the HECT ubiquitin ligase AIP4. Nat Immunol 2009; 10: 1300-1308.

38 Kim MJ, Hwang SY, Imaizumi T, Yoo JY. Negative feedback regulation of RIG-I-mediated antiviral signaling by interferon-induced ISG15 conjugation. J Virol 2008; 82: 1474-1483.

39 Shi HX, Yang K, Liu X, Liu XY, Wei B, Shan YF et al. Positive regulation of interferon regulatory factor 3 activation by Herc5 via ISG15 modification. Mol Cell Biol 2010; 30: 2424-2436.

40 Lu G, Reinert JT, Pitha-Rowe I, Okumura A, Kellum M, Knobeloch $\mathrm{KP}$ et al. ISG15 enhances the innate antiviral response by inhibition of IRF-3 degradation. Cell Mol Biol (Noisy-le-grand) 2006; 52: $29-41$.

Supplementary Information for this article can be found on the Cellular \& Molecular Immunology website (http://www.nature.com/cmi) 